\title{
THE MODULATED E-SPLINE WITH MULTIPLE SUBBANDS AND ITS APPLICATION TO SAMPLING WAVELET-SPARSE SIGNALS
}

\author{
Yingsong Zhang, Pier Luigi Dragotti* \\ Imperial College London
}

\begin{abstract}
The theory of Finite Rate of Innovation (FRI) can be applied to sampling and reconstructing certain classes of parametric signals. The objective of this paper is to have a sub-Nyquist sampling scheme for continuous-time wavelet-sparse signals within the general framework of FRI theory. Though the signal has a parametric representation in the wavelet basis, it is not possible to recover the signal merely from its low-pass samples, which makes the problem different from the conventional FRI settings. The need for the Fourier coefficients at frequencies widely spread over the spectrum puts challenges on the design of the sampling kernel. This paper presents a new family of sampling kernels that are able to stably reproduce exponentials over a wide range of frequencies and gives numerical examples on applying the new kernel to sampling wavelet-sparse signals.
\end{abstract}

Index Terms - FRI, sub-Nyquist sampling, compressive sensing (CS), sparse, $\ell_{1}$ minimization

\section{INTRODUCTION AND PROBLEM SETUP}

Under the FRI framework, an analogue signal is sampled by the ADC unit as shown in Fig. 1, where the discrete samples at the output are

$$
\mathrm{y}_{k}=\langle x(t), \varphi(t / T-k)\rangle .
$$

The sampling kernel $\varphi(t)$ needs to be properly designed so that we can reconstruct the input signal from the discrete samples.

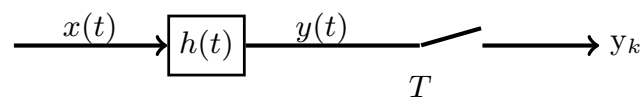

Fig. 1. The structure of FRI sampling where $h(t)=\varphi(-t / T)$.

A few different types of kernels have been proposed by different research groups [1, 2, 3, 4], among which we are particularly interested in the family of exponential reproducing kernels. The elementary member of this family is the E-spline. An E-spline $\beta_{\boldsymbol{\alpha}}(t)$ is any function with Fourier transform

$$
\hat{\beta}_{\boldsymbol{\alpha}}(w)=\prod_{n=0}^{P} \frac{1-e^{\alpha_{n}-j w}}{j w-\alpha_{n}},
$$

and it can reproduce exponential $e^{\alpha_{n} t}$, i.e. there exist weights $c_{n, k}$ such that

$$
\sum_{k \in \mathbb{Z}} c_{n, k} \beta_{\boldsymbol{\alpha}}(t-k)=e^{\alpha_{n} t}
$$

Here $\boldsymbol{\alpha}=\left[\alpha_{0}, \alpha_{1}, \ldots, \alpha_{P}\right]^{T}$ is the column vector containing the generating parameters. Moreover, note that $\alpha_{n}$ could be a real or

\footnotetext{
*This work is supported by the European Research Council (ERC) start-
} ing investigator award Nr. 277800 (RecoSamp). complex number. However, in this paper, we only consider the special case where $\alpha_{n}$ is purely imaginary, i.e. $\alpha_{n}=j w_{n}$ with $w_{n}$ being real. Therefore, for the rest of the paper we use

$$
\boldsymbol{\alpha}=j\left[w_{0}, w_{1}, \ldots, w_{n}\right]^{T}=j \boldsymbol{\omega} .
$$

By definition, the E-spline satisfies the generalized Strang-Fix conditions [5], i.e.

$$
\hat{\beta}_{\boldsymbol{\alpha}}\left(j w_{n}\right) \neq 0 \text { and } \hat{\beta}_{\boldsymbol{\alpha}}\left(j w_{n}+j 2 \pi l\right)=0(\forall l \neq 0),
$$

where $\hat{\beta}_{\boldsymbol{\alpha}}(\cdot)$ represents the Fourier transform of $\beta_{\boldsymbol{\alpha}}(t)^{1}$. The StrangFix conditions (5) are the sufficient and necessary conditions for $\beta_{\boldsymbol{\alpha}}(t)$ to be able to reproduce exponentials and one can show that [4]

$$
c_{n, k}=c_{n, 0} e^{j w_{n} k} \text { with }\left|c_{n, 0}\right|=\frac{1}{\left|\hat{\beta}_{\boldsymbol{\alpha}}\left(j w_{n}\right)\right|} .
$$

For the sake of the conciseness of notation, from now on we use $\hat{\beta}_{\boldsymbol{\omega}}(w)$ instead of $\hat{\beta}_{\boldsymbol{\alpha}}(j w)$.

Using purely imaginary generating parameters allows us to estimate the Fourier transform of $x(t)$ at $j w_{n} / T$. Indeed, once we have sampled the input signal $x(t)$ with the exponential reproducing kernel $h(t)=\beta_{\boldsymbol{\alpha}}(-t / T)$, we obtain the Fourier transform at $j w_{n} / T$ as follows

$$
\begin{aligned}
s_{n} & =\sum_{k \in \mathbb{Z}} c_{n, k} \mathrm{y}_{k}=\sum_{k \in \mathbb{Z}} c_{n, k}\left\langle x(t), \beta_{\boldsymbol{\alpha}}(t / T-k)\right\rangle \\
& =\left\langle x(t), \sum_{k \in \mathbb{Z}} c_{n, k} \beta_{\boldsymbol{\alpha}}(t / T-k)\right\rangle=\left\langle x(t), e^{j \frac{w_{n}}{T} t}\right\rangle .
\end{aligned}
$$

Thus, $s_{n}$ is the Fourier transform of $x(t)$ at frequency $\frac{w_{n}}{2 \pi T}$. This convenient relationship between the discrete spatial samples and the Fourier samples is very useful in designing schemes for the sampling and reconstruction of FRI signals. It has also been used to locate short pulses in ultrasound imaging [3] and on neurophysiology data [6]. These works make the assumption that the pulses all have identical shapes and have significant low-pass components, so that their problem becomes retrieving the location of Diracs from low-pass Fourier coefficients [2].

In this paper, we are interested in sampling sequences of pulses with unknown shape. While the shape is unknown, we assume to know that it is sparse in the wavelet domain. This type of continuoustime sparse signals cannot be recovered by conventional FRI methods, because the signal is a mixture of low-pass-like scaling functions and band-pass-like wavelets. In compressive sensing (CS) a finite-dimensional wavelet sparse signal can be recovered by $\ell_{1}$ minimization from incomplete Fourier information spanning over its whole spectrum [7] (see [8] for the infinite-dimensional extension).

The aim of this paper is to have a realistic and physically realizable acquisition scheme as in Fig. 1 at the same time able to yield Fourier samples at randomly picked frequencies so as to be

\footnotetext{
${ }^{1}$ This is the special case when $\boldsymbol{\alpha}$ is purely imaginary; otherwise, we should use the Laplace transform.
} 


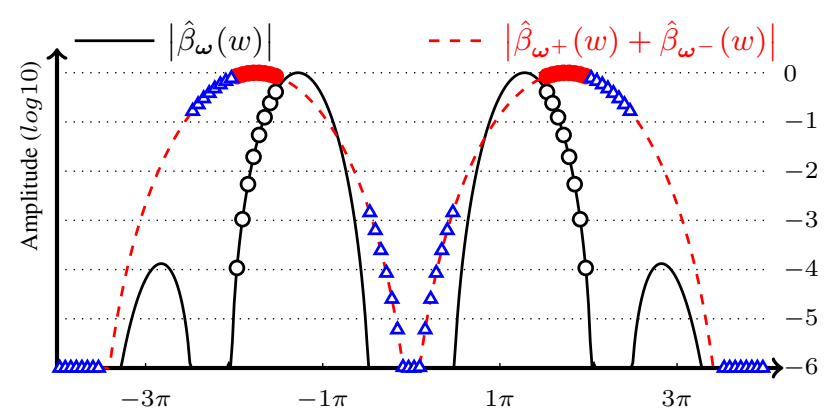

Fig. 2. Illustration of the stability problem of the E-spline. The conventional E-spline $\beta_{\boldsymbol{\omega}}(t)$ reproduce exponential $e^{j w t}$ with $w \in$ $\boldsymbol{w}=\left\{\frac{ \pm k}{32} \mid k=49: 2: 63\right\}$, and the frequency amplitudes at these frequencies are shown by black circles. The red dashed line is used to show the summation of two conventional E-splines with $\hat{\beta}_{\boldsymbol{\omega}}\left(w^{+}\right)$reproducing exponentials with positive parameters from $\boldsymbol{\omega}$ and $\hat{\beta}_{\boldsymbol{\omega}}\left(w^{+}\right)$for the negative parameters. Red solid dots mark $\left|\hat{\beta}_{\boldsymbol{\omega}^{+}}(w)+\hat{\beta}_{\boldsymbol{\omega}^{-}}(w)\right|$ at frequencies $\boldsymbol{\omega}^{ \pm}$. Blue triangles mark $\left|\hat{\beta}_{\boldsymbol{\omega}^{+}}(w)+\hat{\beta}_{\boldsymbol{\omega}^{-}}(w)\right|$ at frequencies $\boldsymbol{\omega}^{ \pm} \pm 2 k \pi$ for some $k \neq 0$. The Strang-Fix conditions are no longer satisfied after summation. Both kernels are scaled so that the maximum frequency amplitude is 1 .

able to use $\ell_{1}$ methods for the reconstruction. We note that conventional CS acquisition techniques cannot achieve this task. The sampling kernels used in FRI cannot be used either, since they cannot stably reproduce exponentials over a wide range of frequencies. To overcome these issues we introduce a new family of exponential reproducing kernels called modulated E-splines that can reproduce exponentials over a wide range of frequencies. We then also introduce a multichannel set-up. Each channel randomly picks a few central frequencies around which a few consequential Fourier samples of the original signals will be obtained, so as to mimic the random distribution.

The paper is organized as follows. Section 2 explains how to design the modulated E-splines. Section 3 presents the multichannel setting. Section 4 shows the numerical examples.

\section{MODULATED REAL E-SPLINE WITH MULTIPLE SUBBANDS}

Because of the Strang-Fix conditions in (5), the essential bandwidth of the sampling device is fixed to be $2 \pi$. To obtain the Fourier samples from different bands, a possible solution is to divide the available bandwidth into parts. We start with the simplest case when the E-spline is like a band-pass filter.

\subsection{A band-pass E-spline}

Take the E-spline shown in Fig. 2 as an example. This E-spline is unstable because the ratio $\frac{\min _{w_{n}}\left|\hat{\beta}_{\omega}\left(w_{n}\right)\right|}{\max _{w_{n}}\left|\hat{\beta}_{\omega}\left(w_{n}\right)\right|}$ (about $10^{-4}$ ) is very small. This is when the noise in the samples creates huge error in generating the Fourier transforms (7).

We use $\boldsymbol{\omega}^{+}$to denote the positive elements of $\boldsymbol{\omega}$ and $\boldsymbol{\omega}^{-}$for the negative elements. According to the definition of the E-spline, we have

$$
\hat{\beta}_{\boldsymbol{\omega}}(w)=\hat{\beta}_{\boldsymbol{\omega}^{+}}(w) \hat{\beta}_{\boldsymbol{\omega}^{-}}(w) .
$$

Both $\hat{\beta}_{\boldsymbol{\omega}^{+}}(w)$ and $\hat{\beta}_{\boldsymbol{\omega}^{-}}(w)$ are radial functions with fast decaying tails, and their centres are far away from each other. The multiplication of such two functions causes the instability issue. For this reason, we consider the summation instead, i.e.

$$
\hat{\psi}_{\boldsymbol{\omega}}(w)=\hat{\beta}_{\boldsymbol{\omega}^{+}}(w)+\hat{\beta}_{\boldsymbol{\omega}^{-}}(w) .
$$

We want that $\hat{\psi}_{\boldsymbol{\omega}}(w)$ be stable and able to reproduce all the exponentials that its addends reproduce. However, we have two problems.

1. $\hat{\psi}_{\boldsymbol{\omega}}\left(w_{n}\right)$ is not necessarily stable even if all of its addends are stable;

2. the Strang-Fix conditions are not necessarily satisfied in this case, as shown in Fig. 2.

Our solution to the above two problems is illustrated by the simplified example in Fig. 3. In this example, there are two addends. The generating parameters of addend 1 is the conjugate of addend 2 , so the summation is a real filter. We want that the two addends act independently on the generating frequencies so that the stability and the Strang-Fix conditions are both maintained. Therefore, we require that addend 1 hits zeros on the frequencies where addend 2 reproduces exponentials (shown by solid dots) and the frequencies where addend 2 requires to be zero; and vice versa. To achieve this, both addends need to be zeros at frequencies whose distance from its generating parameters is a multiple of $\pi$. A conventional E-spline does not satisfy this condition (it is zero at distance multiple of $2 \pi$ ), so we introduce the dilated E-spline

$$
\hat{\beta}_{\omega, 2 M}(w)=\prod_{n=0}^{P} \frac{1-e^{2 M j\left(w_{n}-w\right)}}{2 M j\left(w-w_{n}\right)},
$$

which satisfies

$$
\hat{\beta}_{\boldsymbol{\omega}, 2 M}\left(w_{n}\right) \neq 0, \hat{\beta}_{\boldsymbol{\omega}, 2 M}\left(w_{n}+l \frac{\pi}{M}\right)=0, \forall l \in \mathbb{Z} \backslash 0 .
$$

In our example, addend 1 is $\hat{\beta}_{\boldsymbol{\omega}, 2}(w)$ and addend 2 is $\hat{\beta}_{-\boldsymbol{\omega}, 2}(w)$. We further set the parameters as $\boldsymbol{\omega}=[7 / 6,9 / 6,11 / 6]^{T} \pi$, so that $-w_{n}+2 l \pi=w_{2-n}-3 \pi+2 l \pi$. Using this condition, one can easily verify that the Strang-Fix conditions for $\pm \boldsymbol{\omega}$ are satisfied.

The idea is summarized by the following theorem.

Theorem 2.1. Let

$$
\hat{\psi}_{\boldsymbol{\omega}, 2 M}(w)=\left(\hat{\beta}_{\boldsymbol{\omega}, 2 M}(w)+\hat{\beta}_{-\boldsymbol{\omega}, 2 M}(w)\right),
$$

where $M$ is a positive integer. Assume

$$
\begin{aligned}
& w_{0} \leq w_{1} \leq \cdots \leq w_{P}, w_{P}-w_{0}<\frac{\pi}{M}, \\
& w_{n}+w_{P-n}=k \frac{\pi}{M}, k \in \mathbb{N} \backslash\{2 M l \mid l \in \mathbb{N} .\}
\end{aligned}
$$

Then $\hat{\psi}_{\boldsymbol{\omega}, 2 M}(w)$ is able to reproduce exponential $e^{ \pm j w_{n} t}$, and is a real function:

$$
\psi_{\boldsymbol{\omega}, 2 M}(t)=2 \beta_{\boldsymbol{\omega}-\frac{k \pi}{2 M}, 2 M}(t) \cos \left(\frac{k \pi}{2 M} t\right) .
$$

The proof of the theorem is omitted due to the lack of space. For convenience, we define

$$
\boldsymbol{\omega}_{0}=\boldsymbol{\omega}-\frac{2 \tilde{k}+K_{0}}{2 M} \pi,
$$

in which $\tilde{k}=\left\lfloor\frac{k}{2}\right\rfloor, K_{0}=\operatorname{rem}(k, 2)$, so that the elements of $\boldsymbol{\omega}$ are symmetrically distributed about the origin. Therefore, (14) can be written as

$$
\psi_{\boldsymbol{\omega}, 2 M}(t)=2 \beta_{\boldsymbol{\omega}_{0}, 2 M}(t) \cos \left(\frac{2 \tilde{k}+K_{0}}{2 M} \pi t\right),
$$

which is a function of parameter $M, \boldsymbol{\omega}_{0}$ and $k$. 


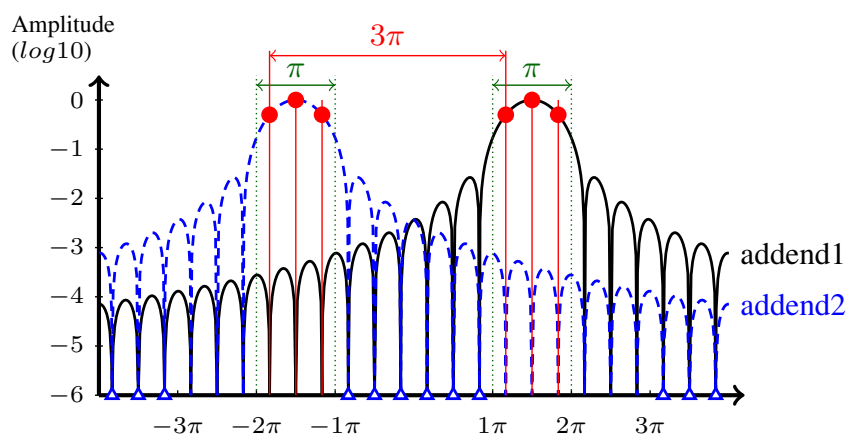

Fig. 3. Illustration for the construction of a band-pass E-spline. addend 1 is the dilated E-spline $\hat{\beta}_{\boldsymbol{\omega}, 2}(w)$ and addend 2 is $\hat{\beta}_{-\boldsymbol{\omega}, 2}$, where $\boldsymbol{\omega}=[7 / 6,9 / 6,11 / 6]^{T} \pi . \hat{\beta}_{\boldsymbol{\omega}, 2}(\boldsymbol{\omega})$ and $\hat{\beta}_{-\boldsymbol{\omega}, 2}(-\boldsymbol{\omega})$ are marked by the solid dots. The two addends act independently on the generating frequencies $\pm \boldsymbol{\omega}$ because addend 1 hits zeros on $-\boldsymbol{\omega}+2 k \pi$ $(\forall k \in \mathbb{Z})$, the frequencies that addend 2 reproduces exponentials at ( $k=0$, shown by solid dots) and the frequencies that addend 2 requires to be zero $(k \neq 0)$ by the Strang-Fix condition; and vice versa. We set $P=2$ here for sake of clarity.

\subsection{Multiple-subband E-spline}

The multiple-subband E-spline is a natural extension of the bandbass E-spline. As illustrated in Fig. 4, we have two band-pass Espline added together. Accordingly, we define, for some real $b_{i}$,

$$
\phi_{\boldsymbol{\omega}_{0}, K_{0}}^{M, N}(t)=\beta_{\boldsymbol{\omega}_{0}, 2 M}(t)\left(\sum_{i=1}^{N} 2 b_{i} \cos \left(\frac{2 k_{i}+K_{0}}{2 M} \pi t\right)\right),
$$

which is the summation of $N$ band-pass E-splines (14). Because (17) is the result of modulating a real E-spline into multiple subbands, we call it modulated E-spline with Multiple subbands (MEMS).

Note that, as the bandwidth of the sampling device is fixed to be $2 \pi$, each addends needs to occupy no more that $2 \pi / N$ bandwidth. Each dilated E-spline occupy $\pi / M$ and every band-pass E-spline is comprised of two dilated E-splines, so that we require $N \leq M$. For the most efficient usage of the bandwidth, we set $N=M$. We use $\hat{\phi}_{\boldsymbol{\omega}_{0}, K_{0}}^{M}$ to denote $\hat{\phi}_{\boldsymbol{\omega}_{0}, K_{0}}^{M, M}$. It is also worth pointing out that the distance between any two addends have to be the multiple of $\frac{\pi}{M}$, namely the bandwidth of each dilated E-spline, in order to satisfy the Strang-Fix condition.

Theorem 2.2. Let $r_{i}=\operatorname{rem}\left(k_{i}, 2 M\right) . \quad K_{0} \in\{0,1\} . \quad M E M S$ $\phi_{\boldsymbol{\omega}, K_{0}}^{M}(t)$ can reproduce exponential $e^{ \pm j w_{n, i} t}$ if

$$
r_{i}+r_{l} \neq 2 M-K_{0} \text { and } \min _{i \neq l}\left|r_{i}-r_{l}\right| \neq 0 .
$$

Here $w_{n, i}=w_{n}+\frac{2 k_{i}+K_{0}}{2 M} \pi$.

Remark 1. The definition of MEMS can be readily generalized to be

$$
\begin{aligned}
\phi_{\boldsymbol{\omega}_{0}, K_{o}}^{M}(t)= & \tilde{\beta}_{\boldsymbol{\omega}_{0}, 2 M}(t) \sum_{i=1}^{N}\left[a_{i} \exp \left(\frac{2 k_{i}+K_{0}}{2 M} \pi t\right)\right. \\
& \left.+b_{i} \exp \left(-\frac{2 k_{i}+K_{0}}{2 M} \pi t\right)\right] .
\end{aligned}
$$

As long as $\tilde{\beta}_{\boldsymbol{\omega}_{0}, 2 M}(t)$ satisfies (11) and $k_{i}$ satisfies (18), one can easily prove that $\phi_{\omega_{0}, K_{o}}^{M}(t)$ can reproduce exponentials $e^{ \pm j w_{n, i} t}$. The generalized MEMS allows us to employ other functions from the E-spline family, for example, we can pre-condition the dilated E-spline as in [4]. Alternatively, we can use a wide range of narrow band-width functions to replace $\tilde{\beta}_{\omega_{0}, 2 M}(t)$, which results in an

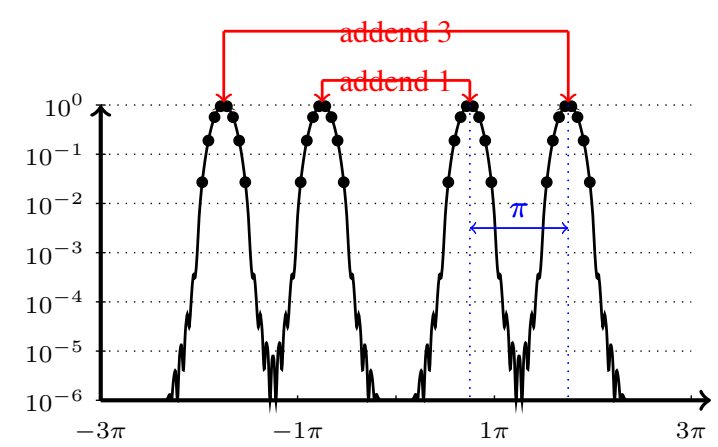

Fig. 4. The frequency amplitude of MEMS, where $M=2, P=$ $7, \boldsymbol{\omega}_{0}=\left\{\frac{k}{32} \mid k=-7: 2: 7\right\}, K_{0}=1, k_{1}=1, k_{2}=3$. $\left|\hat{\phi}_{\boldsymbol{\omega}_{0}, K_{0}}^{M}\left(w_{n}+\frac{2 k_{i}+K_{0}}{4} \pi\right)\right|$ is marked by the solid dot.

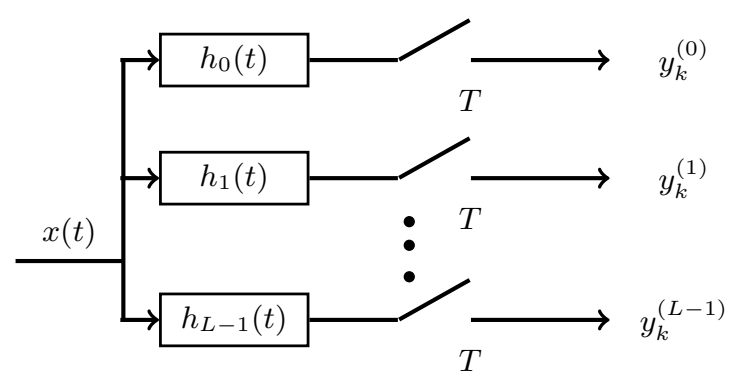

Fig. 5. The multichannel sampling scheme.

approximate Strang-Fix kernel. The quality of such construction depends on how well the Strang-Fix conditions are approximated. See [9] for more details.

\section{SAMPLING THE WAVELET-SPARSE SIGNAL}

We will consider the sampling problem of the wavelet-sparse signal $x(t) \in L_{2}(\mathbb{R})$. We use a vector $\boldsymbol{\eta}$ to denote the wavelet coefficients of $x(t)$. The relationship between the Fourier transform of $x(t)$ at frequency $w$ and $\boldsymbol{\eta}$ can be written as:

$$
\hat{x}(w)=\boldsymbol{\gamma}^{T}(w) \boldsymbol{\eta} .
$$

where $\gamma^{T}(w)$ is a column matrix (refer to [8] for the specific form of $\gamma^{T}(w)$ ). We use $\Omega$ to denote the set of the frequency locations on which $\hat{x}(w)$ are obtained.

Recovering a sparse $\boldsymbol{\eta}$ from its incomplete frequency information is a typical problem in CS. In a nutshell, the CS theory $[8,7,10]$ tells us that 1 ) the member of $\Omega$ needs to be uniformly picked from $-N_{0} / 2 \tau$ to $N_{0} / 2 \tau$, where $N_{0}$ is a constant dependent on the signal, 2 ) the signal can be recovered by the $\ell_{1}$ minimization. We will use MEMS to design a sampling scheme that provides the Fourier coefficients $\hat{x}(\Omega)$ that are almost uniformly distributed along the frequency domain $\left(-\frac{N_{0}}{2 \tau}, \frac{N_{0}}{2 \tau}\right)$, so that we can apply the CS method to reconstruct the signal.

Consider a multichannel scheme with $L$ channels as depicted in Figure 5, and each channel is dedicated to one specific frequency band as shown in Table 1. In each channel, we filter the signal with real $h_{l}(t)$ and sample the filtered signal at interval $T$. The reason for using a multichannel design is to introduce extra flexibility in sampling similar signals without changing the structure, and also to counteract the constrain (18) to better approximate randomness. The sampling rate can be different across the channels. 
Table 1. The sampling scheme for wavelet-sparse signals

\begin{tabular}{c|c|c}
\hline & description & covered frequency band \\
\hline$h_{0}(t)$ & low-pass & $\frac{N_{T}}{N_{0}}(0,1)$ \\
\hline$h_{1}(t)$ & band-pass & $\frac{N_{T}}{N_{0}}(1,2)$ \\
\hline$h_{l}(t)$ & band-pass & $\frac{N_{T}}{N_{0}}\left(2^{l-1}, 2^{l}\right)$ \\
\hline \multicolumn{2}{l}{ Number of channels $L$} & $2^{L-1}<\frac{N_{0}}{N_{T}} \leq 2^{L}$ \\
\hline
\end{tabular}

Note: by default, every channel also covers the negative frequency band symmetric to the positive frequency band described by the table.

Low-pass setting. The objective of the low-pass channel is to produce the low-pass Fourier coefficients at frequencies

$$
\Omega_{0}=\left\{ \pm \frac{2 \pi}{N_{0}} f \mid f=0.5 \ldots, \frac{N_{T}}{2}-0.5\right\},
$$

and this can be achieved by using, for example, the e-MOMs [4] generated by parameters

$$
\alpha=\left\{j \frac{2^{S}}{N_{T}} 2 \pi f, f= \pm 0.5, \ldots, \pm\left(\frac{N_{T}}{2}-0.5\right)\right\} .
$$

Band-pass setting. The objective is that each band-pass channel can reproduce the Fourier coefficients $\hat{x}\left(\Omega_{l}\right)$ where members of $\Omega_{l}$ are randomly picked. For each channel, $h(-t)=\phi_{\omega_{0}, K_{0}}^{M}(t / T)$, and $\phi_{\omega_{0}, K_{0}}^{M}(t)$ is generated by the following parameters.

$$
\begin{aligned}
& P=N_{T} /(2 M)-1 \\
& \boldsymbol{\omega}_{0}=\left\{\frac{2 n-P}{2 P+2} \frac{\pi}{M}, n=0, \ldots, P\right\} \\
& K_{0}=1 ; k_{i}=2 M d_{i}+r_{i}
\end{aligned}
$$

$d_{i}$ is a random integer in set $D_{l}^{2}$, where

$$
D_{l}=\left\{n: n \in \mathbb{Z} \cap\left\lfloor 2^{l-2}\right\rfloor \leq n<\left\lfloor 2^{l-1}\right\rfloor\right\} .
$$

When $l=1, r_{i}$ is a random non-repeated number between $[M, 2 M-1]$, otherwise $r_{i}$ satisfies (18).

The frequencies picked by the band-pass setting is

$$
\Omega_{l}=\left\{\left( \pm \frac{2 n+1}{2 P+2}+k_{i}\right) P \frac{\pi}{N_{0}} \mid n=0, \ldots, P, i=1, \ldots, M_{l}\right\} .
$$

Please note that because $r_{i}$ needs to obey (18) and $\boldsymbol{\omega}_{0}$ is set to pick consecutively $P+1$ frequencies, the members of $\Omega_{l}$ are not truly uniformly chosen from the whole spectrum. However, the distribution of the members of $\Omega_{l}$ is very close to the uniform distribution by choosing a small $P$ and using many channels.

\section{NUMERICAL EXAMPLE}

We will demonstrate the proposed sampling design with a numerical example. The signal, $x(t)$, has 13 non-zero coefficients on the db4 wavelet basis with $J=7$. The support of $x(t)$ is set to be $\tau=1024$ and the finest level is 1 , so that we only need to sample up to $N_{0}=1024$ (the signal can be represented by 1024 discrete samples). The number of nonzeros for each subband is generated randomly but preferring the coarser subband (to mimic the real world signal). For each subbands, the position of the nonzeros are randomly picked and the amplitudes are random numbers. White noise is then added before the signal is sampled by the multichannel system as described in the previous section. We use (7) to obtain the
Table 2. The recovery error of different designs in the presence of noise. The average performance of 100 realizations.

\begin{tabular}{cccccc}
\hline \hline & $50 \mathrm{~dB}$ & $40 \mathrm{~dB}$ & $30 \mathrm{~dB}$ & $20 \mathrm{~dB}$ & $10 \mathrm{~dB}$ \\
\hline Proposed & 0.0025 & 0.0078 & 0.0222 & 0.0591 & 0.1082 \\
Benchmark & 0.0025 & 0.0080 & 0.0245 & 0.0679 & 0.1277 \\
\hline
\end{tabular}

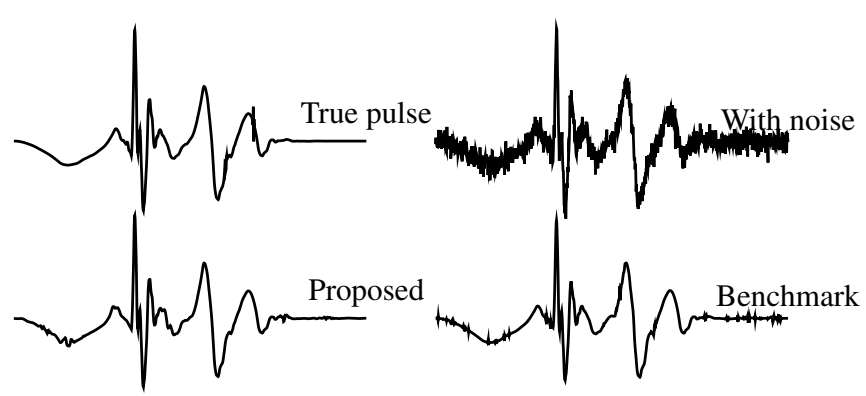

Fig. 6. The comparison between the true pulse and the recovered pulses under the noisy condition (10dB). For each sampling scheme, one recovery result is shown. The recovery is quite faithful to the true pulse shape, though some high-frequency features are missing due to the strong noise.

Fourier coefficients $\hat{x}\left(\Omega_{l}\right)$ from the samples. Finally, we recover the pulse from the Fourier coefficient at $\Omega=\cup_{l=0}^{L} \Omega_{l}$ by solving

$$
\begin{aligned}
& \min |\boldsymbol{\eta}|_{1} \\
& \text { s.t. }\|\hat{x}(\Omega)-\gamma(\Omega) \boldsymbol{\eta}\|_{2} \leq \sigma,
\end{aligned}
$$

where $\sigma$ is parameters related to the noise variance. We denote the estimate as $\tilde{x}(t)$. The relative recovery error is calculated by

$$
R E(\mathrm{x}, \tilde{\mathrm{x}})=\frac{\|x(t)-\tilde{x}(t)\|_{2}}{\|x(t)\|_{2}} .
$$

For each noise level, we randomly generate 100 different $x(t)$ with random white noise. For each $x(t), d_{i}$ and $r_{i}$ are picked according to the instruction given in Section 3 (23)-(24). The proposed methods uses a 6-channel $(L=6)$ setting and $N_{T}=32$ and $\sharp\left(\Omega_{l}\right)=32$. There are total 192 Fourier values.

We use the performance of the ideal random CS setting as the benchmark, where the frequencies in $\Omega_{l}(l>0)$ are uniformly and randomly picked. In the benchmark, we keep the low frequencies in $\Omega_{0}$ fully sampled because that the signal has a significant contribution from the low pass frequencies and this preference for the low frequencies generates much better performance.

In Tab. 2 we show the average performance of the proposed sampling design along with the benchmark, when different levels of noise are added to $x(t)$ before sampling. For the majority of cases with mild noise, our design and the benchmark perform almost equally well. In our experiment, our design performs better than the benchmark in the high-noise cases.

\section{DISCUSSION AND CONCLUSION}

This paper presents a new sampling kernel, MEMS. MEMS is able to reproduce exponentials at frequencies over a wide range, so that the discrete spatial samples from a conventional sampling device with MEMS as sampling kernels can be used to reproduce the Fourier coefficients at either high frequencies or low frequencies. This convenient relationship provides us a way to easily obtain the Fourier coefficients of any signal without the help of specialized equipments. In our numerical example, we have shown that by using properly designed kernels, we can obtain the Fourier information for faithfully reconstructing the wavelet-sparse signal with $\ell_{1}$ minimization. 


\section{REFERENCES}

[1] M. Vetterli, P. Marziliano, and T. Blu, "Sampling signals with finite rate of innovation," Signal Processing, IEEE Transactions on, vol. 50, no. 6, pp. 1417-1428, 2002.

[2] P.L. Dragotti, M. Vetterli, and T. Blu, "Sampling moments and reconstructing signals of finite rate of innovation: Shannon meets strang-fix," Signal Processing, IEEE Transactions on, vol. 55, no. 5, pp. 1741-1757, 2007.

[3] R. Tur, Y.C. Eldar, and Z. Friedman, "Innovation rate sampling of pulse streams with application to ultrasound imaging," Signal Processing, IEEE Transactions on, vol. 59, no. 4, pp. 1827-1842, 2011.

[4] J.A. Urigüen, P.L. Dragotti, and T. Blu, "On the exponential reproducing kernels for sampling signals with finite rate of innovation," in Proceedings of the Sampling Theory and Application Conference, Singapore, 2011.

[5] I. Khalidov, T. Blu, and M. Unser, "Generalized 1-spline wavelet bases," in Optics \& Photonics 2005. International Society for Optics and Photonics, 2005, pp. 59140F-59140F.

[6] J. Oñativia, S. R. Schultz, and P. L. Dragotti, "A finite rate of innovation algorithm for fast and accurate spike detection from two-photon calcium imaging," Journal of neural engineering, vol. 10, no. 4, pp. 046017, 2013.

[7] E.J. Candès, J. Romberg, and T. Tao, "Robust uncertainty principles: Exact signal reconstruction from highly incomplete frequency information," Information Theory, IEEE Transactions on, vol. 52, no. 2, pp. 489-509, 2006.

[8] B. Adcock and A. C. Hansen, "Generalized sampling and infinite-dimensional compressed sensing," Preprint, 2011.

[9] J.A. Urigen, T. Blu, and P.L. Dragotti, "FRI sampling with arbitrary kernels," Signal Processing, IEEE Transactions on, vol. 61, no. 21, pp. 5310-5323, 2013.

[10] E.J. Candès and T. Tao, "Near-optimal signal recovery from random projections: Universal encoding strategies?," IEEE Transactions on Information Theory, vol. 52, no. 12, pp. 5406$5425,2006$. 